\title{
Reproductive Medicine
}

National Cancer Institute

\section{Source}

National Cancer Institute. Reproductive Medicine. NCI Thesaurus. Code C17748.

A branch of medicine that deals with prevention, diagnosis and management of reproductive problems. It addresses issues of sexual education, puberty, family planning, birth control, infertility, reproductive system disease and sexual dysfunction. 